\title{
Correction to: A novel classification and its clinical significance in Chiari I malformation with syringomyelia based on high-resolution MRI
}

\author{
Jian Guan ${ }^{1}$. Chenghua Yuan ${ }^{1}$. Can Zhang ${ }^{1}$ - Longbing Ma ${ }^{1}$ - Qingyu Yao ${ }^{1}$ Lei Cheng ${ }^{1} \cdot$ Zhenlei Liu $^{1}$ - Kai Wang ${ }^{1}$. \\ Wanru Duan ${ }^{1} \cdot$ Xingwen Wang $^{1} \cdot$ Zuowei Wang $^{1} \cdot$ Hao Wu $^{1} \cdot$ Zan Chen $^{1} \cdot$ Fengzeng Jian ${ }^{1}$ (ID
}

Published online: 10 April 2021

(c) Springer-Verlag GmbH Germany, part of Springer Nature 2021

\section{Correction to: European Spine Journal https://doi.org/10.1007/s00586-021-06746-y}

Unfortunately, the Figs. 3 and 5 have been incorrectly published in the original publication. The complete correct Figs. 3 and 5 are given below.

The original article has been corrected.

Publisher's Note Springer Nature remains neutral with regard to jurisdictional claims in published maps and institutional affiliations.

The original article can be found online at https://doi.org/10.1007/ s00586-021-06746-y.

Zan Chen

chenzan66@163.com

$\triangle$ Fengzeng Jian

jianfengzeng@xwh.ccmu.edu.cn

1 Department of Neurosurgery, Division of Spine, China, International Neurological Institute, Xuanwu Hospital, Capital Medical University, 45 Changchun Street, Beijing 100053, People's Republic of China 

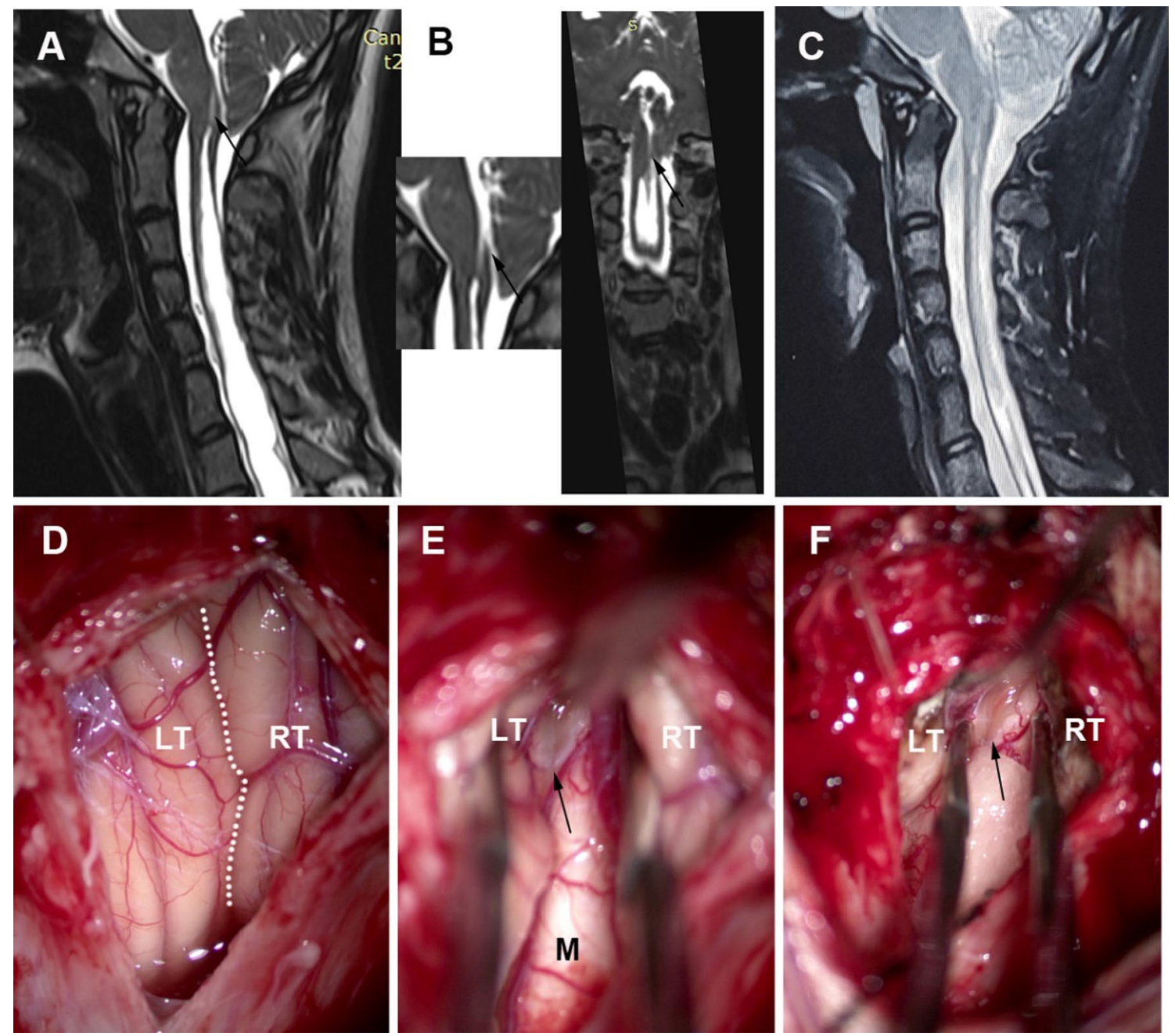

Fig. 3 Partial communicating SM case. a Preoperative midsagittal T2-weighted MR image of CVJ. Note the SM extend upwards to the medulla oblongata without dilated fourth ventricle. b High resolution MR showed a detectable channel between the fourth ventricle and the SM (black arrow) on a certain level. c Postoperative MRI showed marked reduction of the SM. d Incision of the dura shows the medialized tonsils, tonsils occupying the foramen magnum and overlying foramen of Magendie. e The foramen of Magendie is visible, and a semitransparent veil (arrow) is observed. f Subpial tonsillectomy was performed and the arachnoid veil (arrow) is removed 

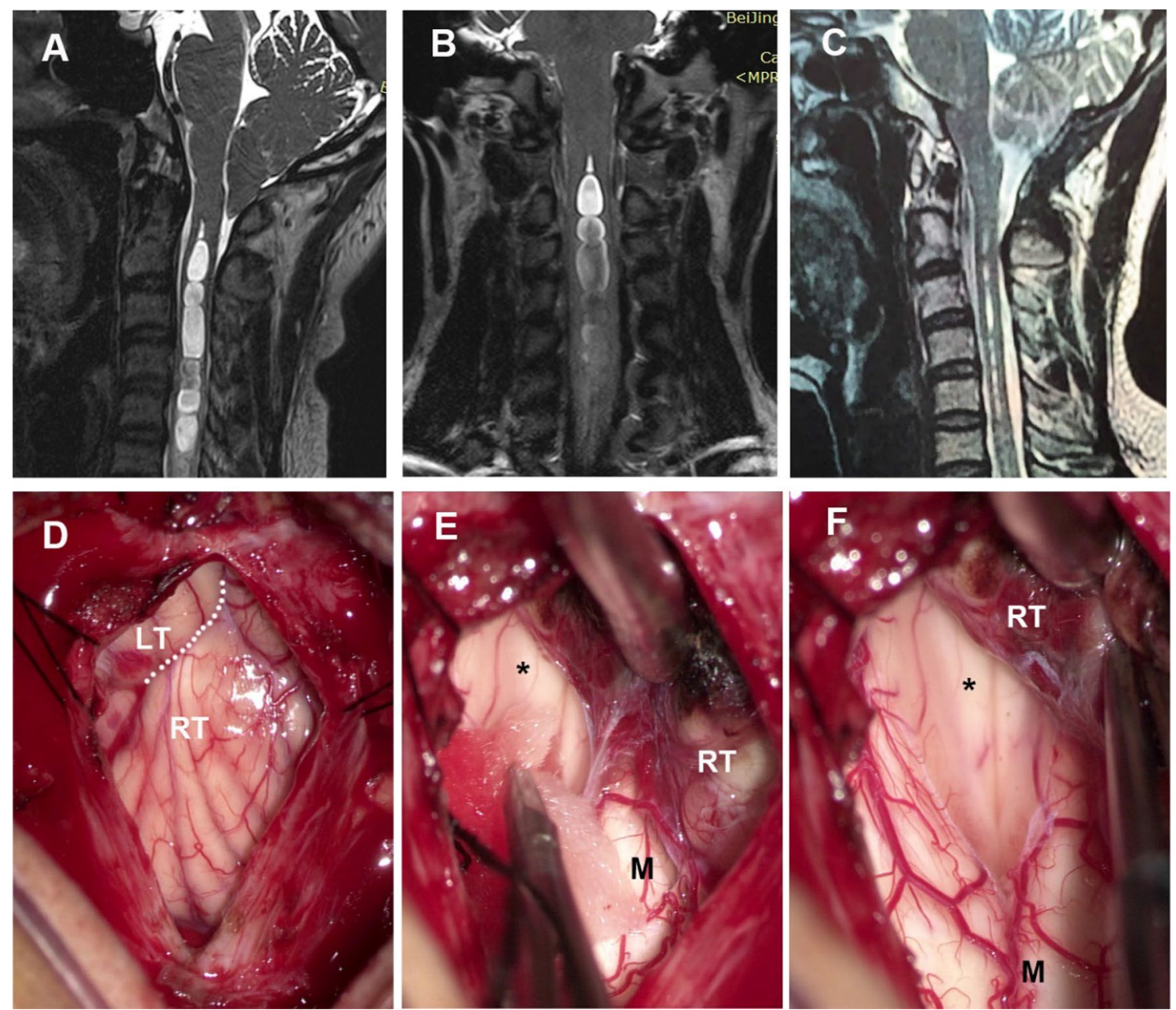

Fig. 5 Non-communicating SM case. a Preoperative midsagittal T2-weighted MR image of CVJ. Note the SM extend upwards to the inferior margin of $\mathrm{C} 1$. b High resolution MR showed the superior extremity of SM does not communicate with the fourth ventricle. c Postoperative MRI showed marked reduction of the SM. d After incision of the dura, it shows the medialized tonsils, tonsils occupying the foramen magnum and overlying foramen of Magendie. $\mathbf{e}$ The foramen of Magendie is uncovered, in which the CSF appears to flow freely (asterisk); f Subpial tonsillectomy was performed and the foramen of Magendie is visible (asterisk). LT left tonsil, M medulla, RT right tonsil 